\title{
THE QUASI-ISOMETRY CLASSIFICATION OF LATTICES IN SEMISIMPLE LIE GROUPS
}

\author{
BENSON FARB
}

\section{Introduction}

This paper is a report on the recently completed quasi-isometry classification of lattices in semisimple ${ }^{1}$ Lie groups. The main theorems stated here are a summary of work of several people over a series of many papers (see below). Our goal here is to give a statement of the final classification, together with a unified discussion (mostly in the form of references to the various pieces), and to pose some related problems. Parts of this work can be viewed as a generalization and strengthening of the Mostow Rigidity Theorem, proven by Mostow, Prasad, and Margulis.

Gromov proposed the program of studying and classifying finitely generated groups up to quasi-isometry. We refer the reader to the papers [Gr1, Gr2, BW] for background and motivation on quasi-isometries. While much of the work has been on invariants, in this paper we will concentrate on rigidity. We will also restrict our attention here to lattices in semisimple groups, and will leave a discussion of rigidity for arbitrary finitely generated groups for a future paper.

Quasi-isometries of lattices. A (coarse) quasi-isometry between metric spaces is a map $f: X \rightarrow Y$ such that, for some constants $K, C, C^{\prime}>0$ :

(1) $\frac{1}{K} d_{X}\left(x_{1}, x_{2}\right)-C \leq d_{Y}\left(f\left(x_{1}\right), f\left(x_{2}\right)\right) \leq K d_{X}\left(x_{1}, x_{2}\right)+C$ for all $x_{1}, x_{2} \in$ $X$.

(2) The $C^{\prime}$-neighborhood of $f(X)$ is all of $Y$.

A map satisfying (1) but not necessarily (2) is called a quasi-isometric embedding. The fundamental group $\Gamma=\pi_{1}(M)$ (endowed with the word metric) of a compact Riemannian manifold $M$ is quasi-isometric to the universal cover $X=\widetilde{M}$. This holds more generally for groups $\Gamma$ acting properly discontinuously and cocompactly by isometries on proper geodesic metric spaces $X$. In particular all uniform lattices in a given Lie group $G$ are quasi-isometric to $G$, hence to each other.

Two quasi-isometries are considered equivalent if they are a bounded distance from each other in the sup norm. Two lattices $\Lambda_{1}, \Lambda_{2}$ in a Lie group $G$ are

Received November 22, 1996.

During work on this paper, the author was supported in part by an N.S.F. postdoctoral fellowship, by I.H.E.S., and by NSF grant \# 9704640.

${ }^{1}$ By semisimple we mean noncompact, connected, semsimple with finite center. By lattice (resp. uniform lattice) we mean a cofinite volume (resp. cocompact) discrete subgroup. 
commensurable if $\left[\Lambda_{2}: g \Lambda_{1} g^{-1} \cap \Lambda_{2}\right]<\infty$ for some element $g \in G$, which is said to commensurate the lattices. Commensurable lattices are clearly quasiisometric.

The following two theorems comprise what we mean by "quasi-isometry classification" of irreducible lattices in semisimple Lie groups among all finitely generated groups. Though many different results are needed to prove these theorems (see below), the main pieces dealing directly with quasi-isometries are essentially due to Pansu [Pa], Schwartz [S1, S2], Farb-Schwartz [FS], Kleiner-Leeb [KL], Eskin-Farb [EF1], and Eskin [E].

Theorem I. [Rigidity of the class of lattices] Let $\Gamma$ be any finitely generated group. If $\Gamma$ is quasi-isometric to an irreducible lattice $\Lambda$ in a semisimple Lie group $G$, then $\Gamma$ is almost a lattice in $G$ : that is, there is a lattice $\Lambda^{\prime}$ in $G$ and a finite group $F$ so that

$$
1 \rightarrow F \rightarrow \Gamma \rightarrow \Lambda^{\prime} \rightarrow 1
$$

is exact.

Theorem II. [Classification among lattices] The quasi-isometry classes of irreducible lattices in semisimple Lie groups are precisely:

- One quasi-isometry class for each semsimple Lie group G, consisting of the uniform lattices in $G$.

- One quasi-isometry class for each commensurability class of irreducible, nonuniform lattice, except in $G=S L_{2}(R)$, where there is precisely one quasi-isometry class of nonuniform lattice.

Remark. Theorems I and II in the case of uniform lattices were announced by Kleiner-Leeb in 1994, and a proof of Theorem II in this case is given in [KL]. They have extended the uniform case of Theorem I to include products with simply connected nilpotent Lie groups. However, the quasi-isometry classification of nilpotent Lie groups remains a major open question.

\section{Remarks.}

(1) Uniform lattices in the same Lie group are quasi-isometric, but often not commensurable. For example one can take uniform lattices whose covolumes are not rationally related (see Chapter 7 of $[\mathrm{Th}]$ for hyperbolic 3-manifold examples).

(2) The previous comment is in marked contrast to the nonuniform case, where nonuniform lattices in $G \neq S L_{2}(R)$ are never quasi-isometric unless they are commensurable. In fact, any quasi-isometry of a nonuniform lattice $\Lambda$ in $G \neq S L_{2}(R)$ is equivalent to a commensurator of $\Lambda$ in $G$. This phenomenon for nonuniform lattices was first discovered by R. Schwartz, in the case of hyperbolic spaces.

(3) Theorems I and II together show that a fixed, nonuniform lattice $\Lambda$ in $G$ is characterized among all finitely generated groups by its quasiisometry type: if $\Gamma$ is any finite generated group quasi-isometric to $\Lambda$, 
then $\Gamma$ itself is (an extension of a finite group by) a nonuniform lattice $\Lambda^{\prime}$ in $G$ commensurable with $\Lambda$.

The proofs of Theorems I and II are given in $\S 3$. The proofs divide into many cases because, as we will see, there are several fundamentally different types of behavior exhibited by different types of lattices. Such behavior can in fact be used to give some qualitative criteria for when certain lattices are "more rigid" than others.

Uniform vs. nonuniform. Unlike uniform lattices, nonuniform lattices $\Gamma$ in $G$ are not quasi-isometric to the symmetric space $X=G / K$ since they do not act cocompactly on $X$. But consider the following construction: chop off every cusp of the quotient $\Gamma \backslash X$ and look at the lifts of each cusp to $X$, giving a $\Gamma$-equivariant union of horoballs in $X$. These horoballs can be made disjoint by cutting the cusp far enough out precisely when $\Gamma$ is so-called $\mathbb{Q}$-rank one (e.g., when $X$ is a hyperbolic or any $\mathbb{R}$-rank one symmetric space, or a product of rank one symmetric spaces). Denote by $\Omega_{\Gamma}$ the symmetric space $X$ with these horoballs deleted, endowed with the path metric. One can also describe $\Omega_{\Gamma}$ as the $\Gamma$-orbit of a lift of any compact, connected subset of $\Gamma \backslash X$. Now $\Gamma$ acts properly discontinuously and cocompactly by isometries on $\Omega_{\Gamma}$, giving a quasi-isometry of $\Gamma$ with this "neutered space" $\Omega_{\Gamma}$.

Thus the geometry of $\Gamma$ lies in the geometry of the associated pattern of deleted horoballs, as well as the symmetric space $X$. It is the more complicated geometry of $\Omega_{\Gamma}$ which allows for the much stronger rigidity of nonuniform lattices.

Historical Remarks. As early as 1982, Gromov pointed out in [Gr1] that the Sullivan-Tukia theorem [Tu1] on uniformly quasiconformal groups implies Theorem I for $G=S O(n, 1)$; this was proved by Cannon-Cooper in [CC] (see Proposition 3.1 below).

The next advance came in Pansu's 1989 paper [Pa], where he proved that every quasi-isometry of quaternionic hyperbolic space of dimension at least two (or of the Cayley hyperbolic plane) is a bounded distance from an isometry, implying Theorem I for uniform lattices $\Lambda$ acting on these spaces (see Proposition 3.1 below). The idea behind Pansu's Theorem is as follows: as Mostow showed, a quasi-isometry $f$ of one of these hyperbolic spaces $X$ induces a map $\partial f$ on the sphere at infinity which is quasiconformal in an appropriate (Heisenberg) sense. A regularity theorem shows that such a map on a sphere is "Heisenberg differentiable" almost everywhere ${ }^{2}$. The differential $D(\partial f)$ gives an automorphism of $N$. Unlike in the real and complex hyperbolic cases, for these Heisenberg groups the automorphism $D(\partial f)$, which is a priori only real-linear, must automatically be quaternionic-linear, so that $\partial f$ is conformal almost everywhere, and so may be extended back into $X$ as an isometry which is a bounded distance from $f$.

\footnotetext{
${ }^{2}$ The Heisenberg group in question is the nilpotent group $N$ in the KAN decomposition of $G=\operatorname{Isom}(X)$. These groups have a one-parameter group of dilations, which can be used to provide a notion of differentiability. See $[\mathrm{Pa}]$.
} 
Though not on lattices in semisimple groups, E. Rieffel's paper [Ri] on uniform lattices in $\operatorname{Isom}\left(H^{2} \times \mathbb{R}\right)$ should also be mentioned.

The first breakthrough in understanding quasi-isometries of nonuniform lattices came with R. Schwartz's 1993 paper [S1] on nonuniform lattices acting on the hyperbolic spaces. He shows that every quasi-isometry of such a lattice is equivalent to a commensurator. The first step uses negative curvature to show that a quasi-isometry $q$ of a neutered space $\Omega_{\Lambda}$ automatically preserves the collection of horosphere boundary components of $\Omega_{\Lambda}$. Schwartz then extends $q$ to a quasi-isometry of the hyperbolic space, and uses an "inversion trick" to show the boundary map is conformal.

For lattices in $G$ with $\operatorname{rank}(G)>1$ new ideas were needed because the curvature of the symmetric space $X=G / K$ is no longer negative, and the associated boundary theory is combinatorial rather than analytic in nature.

The first result for lattices in higher rank groups was by Farb-Schwartz in [FS], who proved every quasi-isometry is equivalent to a commensurator for the classical Hilbert modular groups $\Gamma_{d}=S L_{2}\left(\mathcal{O}_{d}\right)$, which are nonuniform lattices in a product of hyperbolic planes. Here coarse metrical versions of theorems from algebraic topology, such as Alexander Duality and the Jordan Separation Theorem, were developed and used to show that a quasi-isometry of $\Omega_{\Gamma_{d}}$ permutes the components of $\partial \Omega_{\Gamma_{d}}$. To replace the usual equivariance assumption of Mostow Rigidity, the idea of a shadow was introduced. Shadows are patterns of geometrically defined objects associated to a Hilbert Modular group $\Gamma_{d}$, and are automatically preserved by a quasi-isometry. It is the pattern of shadows that allows for reconstruction of the algebraic structure of $\Gamma_{d}$. The coarse topology above, together with a striking result about the rigidity of semisimple abelian group actions on $\mathbb{Z}^{n}$ (which Schwartz calls "Action Rigidity"), generalizes this to $S L_{2}(\mathcal{O})$, where $\mathcal{O}$ is the ring of integers in a number field.

The next advance was by Kleiner-Leeb [KL], who used Gromov's asymptotic cone construction $\operatorname{Con}(X)$ to show that any quasi-isometry $q$ of an irreducible symmetric space $X$ of rank at least two is equivalent to an isometry, a fact which was conjectured by Margulis in the mid 1970's. The idea is that $q$ induces a bilipshitz homeomorphism of $\operatorname{Con}(X)$, which is a certain (nondiscrete) Euclidean building. They then show that any homeomorphism of such a building must be a homothety. A scaling argument allows them to transfer this information back to $X$, and together with ideas from Mostow's proof, shows that such a map on $C o n(X)$ can only come from (up to a bounded amount) an isometry. They also use this method to show that a quasi-isometry of any noncompact globally symmetric space must preserve irreducible factors.

A different, and effective, proof of Margulis's conjecture was then given by Eskin-Farb [EF1]. In this paper, geometric and coarse topological methods were used to show that any quasi-flat in $X$, i.e. a quasi-isometric embedding $\phi$ : $\mathbb{R}^{\operatorname{rank}(X)} \rightarrow X$, lies in a bounded neighborhood of a finite union of maximal 
flats in $X^{3}$, generalizing the fact that a quasigeodesic in a hyperbolic space lies a bounded distance from a geodesic. We note that if $\operatorname{rank}(X)>1$, then there are quasi-flats in $X$ which do not lie in a bounded neighborhood of a single flat. The quasi-flats theorem, together with some ideas from Mostow's proof [Mo1], implies that every quasi-isometry of $X$ is equivalent to an isometry.

The result on quasi-flats proved in [EF1] actually holds with much weaker hypotheses: the map $\phi$ need not be defined on a collection of "holes" of measure proportional to their distance to the origin. In [E], Eskin applies this fact in his proof that every quasi-isometry is a commensurator for nonuniform lattices $\Lambda$ in higher rank symmetric spaces. Using Moore ergodicity and basic ergodic theory, he shows that almost all flats in $X$ have the property that they leave the neutered space $\Omega_{\Lambda}$ precisely in the type of holes mentioned above. A theorem of Lubotzky-Mozes-Raghunathan says that $\Omega_{\Lambda} \rightarrow X$ is a quasi-isometry, so he can apply the "quasi-flats with holes" theorem to (nearly) obtain an automorphism on the Tits boundary of $X$. With more work the proof is completed.

Finally, we would like to mention that several of the themes and techniques in the above work have been extended beyond the realm of lattices in Lie groups (see [FM1, FM2, KaL1, KaL2], for example), although many new phenomena also occur.

\section{Quasi-isometry groups}

For any metric space $X$, one can form the group $Q I(X)$ of all self quasiisometries of $X$ modulo those that are equivalent to the identity. Inverses in $Q I(X)$ exist because quasi-isometries have coarse inverses.

It is easy to check that $Q I(X)$ has the structure of a topological group in the compact-open (= uniform convergence on compacta) topology. For finitely generated groups $\Gamma$ endowed with the word metric, the group $Q I(\Gamma)$ is independent of the choice of generators for $\Gamma$. More generally, a quasi-isometry $f: X \rightarrow Y$ induces an isomorphism $f_{*}: Q I(X) \rightarrow Q I(Y)$ of topological groups.

Much of the work in proving Theorems I and II comes from computing quasiisometry groups of lattices. We will often implicitly use the fact that a uniform lattice $\Lambda$ in $G$ is quasi-isometric to both $G$ and to the symmetric space $X=$ $G / K$. We now list the computations of the quasi-isometry groups of irreducible lattices in semisimple Lie groups. For details of the proofs of the theorems quoted below, we refer the reader to the original papers.

2.1. Uniform lattices. For a uniform lattice $\Lambda$ acting on the symmetric space $X$, the quasi-isometry group $Q I(\Lambda)$ is clearly isomorphic to $Q I(X)$, so it suffices to compute the latter group.

- Real and complex hyperbolic spaces. For $n>1$, the quasi-isometry group of $X=\mathbb{R} \mathbb{H}^{n+1}$ or $X=\mathbb{C} \mathbb{H}^{n}$ is precisely the (Carnot) quasiconformal group of the sphere at infinity $\partial X$. Mostow [Mo2] (see also

\footnotetext{
${ }^{3} \mathrm{An}$ independent proof of this result was given by Kleiner-Leeb using different methods, and is in the latest version of $[\mathrm{KL}]$.
} 
[GP]) showed that every quasi-isometry of $X$ induces a quasiconformal homeomorphism of $\partial X$, and that two quasi-isometries of $X$ are equivalent if and only if they induce the same map on $\partial X$.

Tukia $\left(X=\mathbb{R H}^{n}\right)$ and Koranyi-Reimann $\left(X=\mathbb{C H}^{n}\right)$ showed (see $[\mathrm{Tu} 4, \mathrm{KR}]$ ) that every (Heisenberg) quasiconformal map $f^{*}: \partial X \rightarrow \partial X$ may be extended to a quasi-isometry $f: X \rightarrow X$ as follows: first define a map $p$ from the space of distinct triples in $\partial X$ into $X$ by defining $p(a, b, c)$ to be the closest point (i.e. orthogonal) projection of $c$ onto the geodesic from $a$ to $b$. Now given $x \in X$, let $(a, b, c)$ be any triple with $p(a, b, c)=x$, and define $f(x)=p\left(f^{*}(a), f^{*}(b), f^{*}(c)\right)$. One then checks that a different choice of $(a, b, c)$ with $p(a, b, c)=x$ changes $f$ only by a bounded amount, and that $f$ is indeed a quasi-isometry. Hence the quasi-isometry group $Q I(X)$ is isomorphic to the group of (Heisenberg) quasiconformal homeomorphisms of $\partial X$. In the case of $\mathbb{H}^{2}$, the quasi-isometry group is the quasi-symmetric group of the circle at infinity, although we will not need this fact.

- Quaternionic hyperbolic spaces and the Cayley hyperbolic plane. As mentioned above, Pansu $[\mathrm{Pa}]$ proved that $Q I\left(\mathbb{Q H}^{n}\right) \approx I \operatorname{som}\left(\mathbb{Q H}^{n}\right)$ for $n>1$ and $Q I\left(\mathbf{C a H}^{2}\right) \approx I \operatorname{som}\left(\mathbf{C a H}^{2}\right)$. In this way these spaces are much more rigid than their real and complex counterparts, and behave more like irreducible, higher rank symmetric spaces.

- Irreducible, higher rank symmetric spaces of noncompact type. In this case Kleiner-Leeb [KL], and later Eskin-Farb [EF1] by a different method, showed that $Q I(X) \approx I \operatorname{som}(X)$.

- Products of symmetric spaces. Kleiner-Leeb [KL] show that a quasiisometry of a product of irreducible symmetric spaces of noncompact type $X_{1} \times \cdots \times X_{n}$ is equivalent to a product of quasi-isometries of the factors, that is $Q I\left(X_{1} \times \cdots \times X_{n}\right) \approx Q I\left(X_{1}\right) \times \cdots \times Q I\left(X_{n}\right)$.

2.2. Nonuniform lattices. In this section we summarize the proof of the following:

Theorem 2.1. (Quasi-isometries are commensurators) Let $\Lambda$ be an irreducible, nonuniform lattice in a semisimple Lie group $G \neq S L_{2}(\mathbb{R})$. Then every quasi-isometry of $\Lambda$ is equivalent to a commensurator.

The proof of this theorem requires a verbatim application of tools from various papers. These tools are due to Schwartz [S1, S2], Farb-Schwartz [FS], and Eskin [E]. Let $X=G / K$ denote the symmetric space associated to $G$.

- $G$ has no rank one factors. This is Theorem 0.1 of $[\mathrm{E}]$.

- $G$ is rank one. This is the main theorem of [S1].

- $G$ is a product of rank one factors, containing at least one $S L_{2}(\mathbb{R})$ factor. The case of $S L_{2}\left(\mathcal{O}_{d}\right)$, where $\mathcal{O}_{d}$ is the ring of integers in a totally real, degree two extension of $\mathbb{Q}$, is the main theorem of [FS]. The case of $S L_{2}(\mathcal{O}), \mathcal{O}$ the ring of integers of a number field, is the 
main theorem of [S2]. In general one applies the Boundary Detection Theorem in [FS] or [S2] and then the Action Rigidity Theorem of [S2].

- $G$ is a product of rank one groups with no $S L_{2}(\mathbb{R})$ factors. By [E], any quasi-isometry $f: \Lambda \rightarrow \Lambda$ extends to a factor-preserving quasiisometry $\hat{f}: X \rightarrow X$. By the Boundary Detection Theorem of [FS, S2], $f$ preserves the collection of horospherical boundary components of the neutered space associated to $\Lambda$. Now apply the zooming argument of [FS], which gives that $\hat{f}$, when restricted to each factor of $X$, has linear boundary values. Since $G$ has no $S L_{2}(\mathbb{R})$ factor, we may apply the Inversion Trick of [S1] factor by factor to give that $\hat{f}$ is a bounded distance from an isometry of $X$. Lemma 8.1 of [E] (due to N. Shah) then gives that $f$ is equivalent to a commensurator.

- $G$ is a product with at least one rank one factor and at least one simple factor $G_{1}$ of rank at least two. As in the previous case, a quasi-isometry $f: \Lambda \rightarrow \Lambda$ extends to a factor-preserving quasi-isometry $\hat{f}: X \rightarrow X$, where $X$ is the symmetric space for $G$. Let $X=Y \times Z$ be the factorization of $X$ into a product of symmetric spaces, where $Z$ is the (irreducible) symmetric space for $G_{1}$. Let $\hat{f}=g \times h$ be the factorization of $\hat{f}$ as a product of quasi-isometries $g$ of $Y$ and $h$ of $Z$. By [KL] or [EF1], $h$ is (equivalent to) an isometry.

Now $\Lambda$ is obtained from restriction of scalars from some absolutely simple group defined over a number field $K$. Let $B$ be the Borel subgroup of $G$. Then $\Lambda$ acts on the $K$-points (a dense subset) of the boundary $G / B$ with finitely many orbits - this is essentially finiteness of cusps.

Since $G$ has at least one rank one factor, we know that $\Lambda$ has $\mathbb{Q}$ rank one. So by the Boundary Detection Theorem in [FS] or [S2], $\hat{f}$ preserves the set of horospherical boundary components of the neutered space associated to $\Lambda$. Hence $\partial \hat{f}$ preserves $K$-points on the boundary. One can conclude that the isometry $h$ is defined over $K$, and so by composing $\hat{f}$ by a commensurator of $\Lambda$ we can assume $h$ is the identity. But $\Lambda$ is irreducible, and $\partial g \times \partial h=\partial g \times I d$ is uniformly continuous by [E]. Hence $\partial g$ is the identity on a dense set of points, hence everywhere, so that $\hat{f}=g \times h$ is equivalent to the identity (after having composed with a commensurator).

\section{Proof of Theorems I and II}

3.1. A rigidity condition. The following is a (now standard) principle due essentially to Cannon-Cooper [CC], and was also indicated by Gromov [Gr1].

For simplicity of notation we call a map $f$ a $K$-quasi-isometry if each of the three constants $K, C, C^{\prime}$ in the definition of quasi-isometry is at most $K$. A group $H<Q I(X)$ is a uniform group of quasi-isometries of $X$ if there exists $K \geq 1$ so that each $f \in H$ is a $K$-quasi-isometry. 
Proposition 3.1. (QI rigidity condition) Let $X$ be a proper geodesic metric space, and let $G=I \operatorname{som}(X)$. Suppose $\Gamma$ is a finitely generated group which is quasi-isometric to $X$. Then there is a homomorphism $\Psi: \Gamma \rightarrow Q I(X)$ with finite kernel and with $\Psi(\Gamma) \backslash X$ compact.

If further $\Psi(\Gamma)$ is conjugate into $G$ (for example if $Q I(X)=G$ ), then $\Gamma$ almost admits a geometric action on $X$ : there is a finite group $F$ and a finitely generated group $\Lambda$ acting cocompactly, properly discontinuously by isometries on $X$ so that

$$
1 \rightarrow F \rightarrow \Gamma \rightarrow \Lambda \rightarrow 1
$$

is exact.

Proof. Let $f: \Gamma \rightarrow X$ be a $K$-quasi-isometry with coarse inverse $f^{-1}$. Left multplication $L_{g}(h)=g h$ is an isometry of $\Gamma$ for each $g \in \Gamma$, so $f \circ L_{g} \circ f^{-1}$ is a $K^{2}$-quasi-isometry of $X$ for each $g \in \Gamma$. The map $\Psi: \Gamma \rightarrow Q I(X)$ given by

$$
\Psi(g)=f \circ L_{g} \circ f^{-1}
$$

is a homomorphism since $d(\Psi(g h), \Psi(g) \circ \Psi(h))<\infty$, and quasi-isometries a bounded distance from each other are identified in $Q I(X)$.

First note that $\Psi(\Gamma)$ acts cocompactly on $X$ since $N b h d_{C}(f(\Gamma))=X$ for some $C>0$.

Now pick a basepoint $x \in X$. Since $\Psi(g)$ was defined via the conjugated action of $\Gamma$, and $f$ is a quasi-isometry, and by the uniformity of everything involved, there is a constant $C^{\prime}>0$ so that $d_{X}(x, \Psi(g) \cdot x) \leq C^{\prime}$ for only finitely many $g \in \Gamma$; in particular this distance is 0 for only finitely many $g \in \Gamma$. From this it follows that $\Psi$ has finite kernel and the action of $\Psi(\Gamma)$ on $X$ is properly discontinuous.

If $q \Psi(\Gamma) q^{-1} \subset G$ for some $q \in Q I(X)$, then the final conclusion clearly holds with $\Lambda=q \Psi(\Gamma) q^{-1}$.

3.2. Proof of Theorem I. The proof falls into several cases, depending on what kind of lattice $\Lambda$ is. The main aspects of most parts of the proof are the computations presented in $\S 3.1$.

- $\Lambda$ is uniform and $G=\operatorname{Isom}\left(\mathbb{H}^{2}\right)$. A group $\Gamma$ of homeomorphisms of $S^{1}$ is a convergence group if, for all sequences $\left\{g_{i}\right\}$ of elements $g_{i} \in \Gamma$, there exist $x, y \in S^{1}$ and a subsequence $\left\{g_{i_{k}}\right\}$ so that $g_{i_{k}} \rightarrow y$ and $g_{i_{k}}^{-1} \rightarrow x$ uniformly on compacta in $S^{1} \backslash\{x, y\}$. The convergence groups conjecture, proved by Casson-Jungreis [CJ] and Gabai [Ga] completing important earlier work of Tukia [Tu2], says that any convergence group is conjugate in $\operatorname{Homeo}\left(S^{1}\right)$ to a Fuchsian group.

Now Mess $[\mathrm{Me}]$ shows that any group $\Gamma$ quasi-isometric to $\mathbb{H}^{2}$ acts on $S^{1}$ with finite kernel, properly discontinuously and cocompactly on the space of distinct triples, and so is a convergence group. Alternatively, since $\Gamma$ is quasi-isometric to $\mathbb{H}^{2}, \Gamma$ is a word-hyperbolic group with 
boundary homeomorphic to $S^{1}$. Word-hyperbolic groups act on their boundaries as convergence groups ([Fr, Tu3]), hence they are conjugate to Fuchsian groups.

- $\Lambda$ is uniform and $G=\operatorname{Isom}\left(H^{n}\right), n>2$ or $G=\operatorname{Isom}\left(C H^{n}\right), n>1$. Here the quasi-isometry group is precisely the (Carnot) quasi-conformal group of the sphere at infinity. In the real case, Sullivan [Su] $(n=3)$ showed that any uniformly quasiconformal group $H$ on $S^{n-1}$ is quasiconformally conjugate to a Möbius group, i.e. subgroup of $G$. This is generally not true for $n>3^{4}$. However, for $n \geq 3$, Tukia [Tu1] proved that if in addition the natural action of $H$ on the space of triples of distinct points of $S^{n-1}$ is cocompact, then $H$ is quasiconformally conjugate to a Möbius group. This same fact holds for the Heisenberg quasiconformal groups which occur as the boundary group in the complex hyperbolic case, as was proved by Chow [Ch] based on Tukia's outline, using the work of Koranyi-Reimann [KR].

We now repeat the proof given by Cannon-Cooper [CC] and Chow [Ch]. If $\Gamma$ is any group quasi-isometric to $\Lambda$, hence to $X=G / K$, the action of $\Gamma$ by a uniform group of quasi-isometries of $X$ given in Proposition 3.1 gives an action by uniformly quasiconformal maps on $\partial X$. Since this action is just the (transitive) action of $\Gamma$ on itself conjugated by a quasi-isometry $f: \Gamma \rightarrow X$, and since $n b h d_{C}(f(\Gamma))=X$ for some $0<C<\infty$, the action of $\Gamma$ is cocompact on $X$. Since the map $p$ from the space of distinct triples in $\partial X$ to $X$ is compact-to-one (cf. the discussion of $Q I\left(\mathbb{H}^{n}\right)$ on page 709$)$, the action of $\Gamma$ on the triple space is also cocompact.

Hence we may conjugate the $\Gamma$-action to a Möbius action, which is to say to an isometric action on $X$. Theorem $I$ then follows by applying Proposition 3.1

- $\Lambda$ is a uniform lattice in $G=\operatorname{Isom}\left(\mathbb{Q H}^{n}\right), n>1$ or $G=\operatorname{Isom}\left(\mathbf{C a H}^{2}\right)$ or $G$ is irreducible with $\operatorname{rank}(G)>1$. In these cases the quasi-isometry group of the symmetric space $X=G / K$, hence of $\Lambda$, equals the isometry group of $X$, and Theorem I follows from Proposition 3.1.

- $\Lambda$ is a uniform lattice in a product $X_{1} \times \cdots \times X_{n}$ of irreducible $X_{i}$. The technique of Proposition 3.1 gives a representation $\Gamma \rightarrow$ $Q I\left(X_{1} \times \cdots \times X_{n}\right)=Q I\left(X_{1}\right) \times \cdots \times Q I\left(X_{n}\right)$ of $\Gamma$ as a uniform group of quasi-isometries. The projection to each $Q I\left(X_{i}\right)$ gives a cocompact and uniform action, so we may apply the arguments above to each projection $\Gamma \rightarrow Q I\left(X_{i}\right)$ to obtain a cocompact and discrete representation $\Gamma \rightarrow \operatorname{Isom}\left(X_{1}\right) \times \cdots \times I \operatorname{som}\left(X_{n}\right)$ with finite kernel. Note that, for $\mathbb{H}^{2}$ factors one can apply the Convergence Groups Theorem, as above, since a uniformly quasisymmetric group on the circle is a convergence group. Alternatively, one may also use Hinkannen's Theorem [Hi], which says

\footnotetext{
${ }^{4}$ Sullivan's proof works only for $n-1=2$ because his proof uses the measurable Riemann mapping theorem in an essential way.
} 
that a nondiscrete, uniformly quasi-symmetric group on $S^{1}$ is quasisymmetrically conjugate to a Mobius group. We remind the reader that every quasi-symmetric map of $S^{1}$ has a quasi-isometric extension to the hyperbolic plane.

- $\Lambda$ is a nonuniform lattice in $G=S L_{2}(R)$. In this case $\Lambda$ is virtually free. A proof that any group $\Gamma$ quasi-isometric to a free group is almost a free group is given by Ghys and de La Harpe ([GH], Theorem 7.19) for torsion-free $\Gamma$. A different proof for any finitely generated $\Gamma$ is given by Gersten in [Ge2]. Here is the idea of the proof in $[\mathrm{GH}]$ : the property of being a word-hyperbolic group, and the topological type of the boundary of a word-hyperbolic group, are easily seen to be quasiisometry invariants, hence $\Gamma$ must be word-hyperbolic with Cantor set boundary, in particular $\Gamma$ is finitely presented. By Stallings' theorem on ends [St] and Dunwoody's Accessibility Theorem, we may split $\Gamma$ as a free product (perhaps amalgamated over finite groups) of a finite number of subgroups with 0,1 , or 2 ends. It is easy to check that each of these subgroups is quasiconvex in $\Gamma$, hence is word-hyperbolic. Since $\partial \Gamma$ is a Cantor set, and since each of these subgroups is a word-hyperbolic group whose boundary embeds into $\partial \Gamma$, the case of one end cannot occur. But groups with 0 ends are finite, and groups with 2 ends are virtually cylic, so $\Gamma$ is a free product of finite and virtually cyclic groups, perhaps amalgamated over finite groups. Hence $\Gamma$ is virtually free.

- $\Lambda$ is nonuniform, irreducible in $G \neq S L_{2}(\mathbb{R})$. As in Proposition 3.1, we may conjugate the left action of $\Gamma$ on itself by the quasi-isometry $q: \Gamma \rightarrow \Lambda$ to get a homomorphism $\rho: \Gamma \rightarrow Q I(\Lambda) \approx \operatorname{Comm}(\Lambda)<G$. The same arguments as in the proof of Proposition 3.1 show that $\rho(\Gamma)$ is discrete, $\rho$ has finite kernel, and $\rho(\Gamma)$ acts cocompactly on $\Omega$. This last fact shows that $\rho(\Gamma)$ acts on $X$ with cofinite volume.

3.3. Proof of Theorem II. As noted above, a quasi-isometry $q: \Gamma_{1} \rightarrow \Gamma_{2}$ induces an isomorphism $q_{*}: Q I\left(\Gamma_{1}\right) \rightarrow Q I\left(\Gamma_{2}\right)$ of topological groups.

Suppose $\Lambda_{1}, \Lambda_{2}$ are two irreducible lattices in semisimple Lie groups $G_{1}, G_{2}$, and that $\Lambda_{1}$ is quasi-isometric to $\Lambda_{2}$. By Theorem I, we may (perhaps by replacing $\Lambda_{1}$ by an extension of $\Lambda_{1}$ by a finite group) assume that $\Lambda_{1}$ and $\Lambda_{2}$ are both lattices in $G_{2}$. By the computations of quasi-isometry groups of lattices presented in $\S 2$, it follows that $\Lambda_{1}$ is uniform if and only if $\Lambda_{2}$ is uniform. Alternatively, as noted in [Ge2], a uniform lattice in $G_{2}$ cannot be quasi-isometric to a nonuniform lattice in $G_{2}$ : By Block-Weinberger [BW] and Gersten [Ge1], virtual cohomological dimension is a quasi-isometry invariant for groups with finite classifying space, and this dimension is different for uniform and nonuniform lattices in the same semisimple Lie group (see, e.g. [Br], Remark 4, p.217).

Now if each $\Lambda_{i}$ is uniform in $G_{2}$, then by the observation of Milnor-Svarc each $\Lambda_{i}$ is quasi-isometric to $G_{2}$, hence to each other. If each $\Lambda_{i}$ is nonuniform, 
then by the computations of quasi-isometry groups of lattices presented in $\S 2$, $\Lambda_{1}$ and $\Lambda_{2}$ are commensurable unless $G=P S L_{2}(\mathbb{R})$, in which case each $\Lambda_{i}$ is virtually free.

\section{Some questions}

We state here three problems concerning quasi-isometries in the context of lattices and symmetric spaces.

Problem A : Every nonuniform lattice in $\operatorname{PSL}(2, \mathbb{R})$ contains an $n$-generator free group $F_{n}, n>1$ of finite index. The group $Q I\left(F_{n}\right)$ may be identified with the group of quasi-symmetric maps of the Cantor set $\partial F_{n}$. Is it true that any uniform group of quasi-isometries of $F_{n}$, perhaps with an extra cocompactness assumption, is conjugate by a quasi-isometry to a group of isometries of $F_{n}$ (here we think of $F_{n}$ as a regular tree of valence $2 n$ )? This would imply a proof of Theorem I for free groups which doesn't use Stallings' theorem on ends, and which is similar in spirit to the proof of Theorem $\mathrm{I}$ in the case of $\mathbb{H}^{n}, n>2$, as follows.

Once we have that our group $\Gamma$ quasi-isometric to $F_{n}$ is conjugate to a group of isometries of $F_{n}$, we are done, since $\operatorname{Isom}\left(F_{n}\right)$ is an extension by $F_{n}$ of the stabilizer in $\operatorname{Isom}\left(F_{n}\right)$ of a point $x \in F_{n}$, which is a profinite group, hence compact.

A related question: since most elements of $Q I\left(F_{n}\right)$ do not come from elements of $P S L_{2}(\mathbb{R})$, in particular $Q I\left(F_{n}\right)$ is much bigger than the commensurator group of any lattice in $P S L_{2}(\mathbb{R})$. Does $Q I\left(F_{n}\right)$ equal the abstract commensurator of $F_{n}$ ?

The following two questions seem to be well-known. Some recent progress on them has been made by Kleiner-Leeb (personal communication).

Problem B : Let $X$ and $Y$ be symmetric spaces of noncompact type. Find necessary and sufficient conditions for $X$ to admit a quasi-isometric embedding into $Y$. One obstruction for such an embedding should be that $\operatorname{rank}(X) \leq$ $\operatorname{rank}(Y)$. Pansu's conformal dimension gives another obstruction in the rank one case (see [Gr2]). But there are also some surprising embeddings, for example $\mathbb{H}^{3}$ quasi-isometrically embeds in $\mathbb{H}^{2} \times \mathbb{H}^{2}$ (see $[\mathrm{BF}]$ ).

Problem C : Suppose $X$ quasi-isometrically embeds in $Y$. If $X$ is not a real hyperbolic space, is it true that every such embedding lies in a bounded neighborhood of an isometric embedding? Note that the usual bending constructions as in $[\mathrm{Th}, \mathrm{JM}]$ give quasi-isometric embeddings $\mathbb{H}^{m} \rightarrow \mathbb{H}^{n}, n>m>1$ whose image does not lie in a bounded neighborhood of an isometric embedding. Further note that superrigidity does not necessarily preclude the existence of "exotic" quasi-isometric embeddings of higher rank symmetric spaces. 


\section{Acknowledgements}

I'd like to express my appreciation to Kevin Corlette and Bob Zimmer for their continued support, encouragement, and many useful discussions on this material. I would also like to thank Rich Schwartz and Alex Eskin, who are responsible for many of the advances summarized here, and with whom I've had the pleasure of collaboration, as well as edifying discussions too numerous to mention. Finally, I gratefully acknowledge the hospitality and financial support of I.H.E.S.

\section{References}

[BF] N. Brady and B. Farb, Filling-invariants at infinity for manifolds of nonpositive curvature, Trans. Amer. Math. Soc., to appear.

[BG] M. Bridson and S. Gersten, The optimal isoperimetric inequality for torus bundles over the circle, Quart. J. Math (Oxford) 2 (1996), 1-24.

[Br] K. Brown, Cohomology of Groups, Grad. Texts in Math., 87, Springer-Verlag, 1982.

[BW] J. Block and S. Weinberger, Large scale homology theories and geometry, Proc. of the Georgia International Topology Conference of 1993, International Press., to appear.

[Ca] J. Cannon, The theory of negatively curved spaces and groups, in Ergodic theory, symbolic dynamics, and hyperbolic spaces, ed. by C. Series T. Bedford, M. Keane, ed., Oxford Univ. Press, 1991.

[CC] J. Cannon and D. Cooper, A charactrization of cocompact hyperbolic and finitevolume hyperbolic groups in dimension three, Trans. Amer. Math. Soc., 330 (1992), 419-431.

[Ch] R. Chow, Groups coarse quasi-isometric to complex hyperbolic space, Trans. Amer. Math. Soc. 348 (1996), 1757-1769.

[CJ] A. Casson and D. Joungreis, Convergence groups and Seifert fibered 3 -manifolds, Invent. Math 118 (1994), 441-456.

[E] A. Eskin, Quasi-isometric rigidity for nonuniform lattices in higher rank, November 1996 preprint (submitted).

[EF1] A. Eskin and B. Farb, Quasi-flats and rigidity in higher rank symmetric spaces, J. Amer. Math. Soc., 10 (1997), 653-692.

[EF2] A. Eskin and B. Farb, Quasi-flats in $\mathbb{H}^{2} \times \mathbb{H}^{2}$, Proc. of the Colloquium on Lie groups and Ergodic Theory, Tata Institute 1996, to appear.

[FM1] B. Farb and L. Mosher (appendix by D. Cooper), A rigidity theorem for the solvable Baumslag-Solitar groups, Invent. Math., to appear.

[FM2] B. Farb and L. Mosher, Quasi-isometric rigidity for the solvable Baumslag-Solitar groups. II, preprint.

[FS] B. Farb and R. Schwartz, The large-scale geometry of Hilbert modular groups, J. Diff. Geom. 44 (1996), 435-478.

[Fr] E. Freden, Negatively curved groups have the convergence property. I, Ann. Acad. Sci. Fenn. Ser. A I Math. 20 (1995), 333-348.

[Ga] D. Gabai, Convergence groups are Fuchsian groups, Ann. of Math. (2) 136 (1992), 447-510.

[Ge1] S. M. Gersten, Quasi-isometry invariance of cohomological dimension, C. R. Acad. Sci. Paris Série 1 Math., 316 (1993), 411-416.

[Ge2] , Isoperimetric functions of groups and exotic cohomology, in Combinatorial and Geometric Group Theory: Edinburgh 1993, Ed. by A. Duncan, N. Gilbert, and J. Howie, LMS Lecture Notes, 204, Cambridge Univ. Press, 1995.

[Gr1] M. Gromov, Infinite groups as geometric objects, in Proceedings of the International Congress of Mathematicians (Warsaw, 1983), pp. 385-392, PWN, Warsaw, 1984. 
[Gr2] _ Asymptotic invariants of infinite groups, Geometric Group Theory (G. Niblo and M. Roller, eds.), LMS Lecture Notes, 182, Cambridge Univ. Press, 1993.

[GH] E. Ghys and P. de la Harpe (eds.), Sur les groupes hyperboliques d'ápres Mikhael Gromov, Progess in Math., 83, Birkhauser, 1990.

[GP] M. Gromov and P. Pansu, Rigidity of lattices: An introduction, Geometric topology: recent developments (Montecatini Terme, 1990), pp. 39-137, Lecture Notes in Math., 1504, Springer, Berlin, 1991

[Hi] A. Hinkkanen, The structure of certain quasisymmetric groups, Mem. Amer. Math. Soc. 83 (1990).

[JM] D. Johnson and J. J. Millson, Deformation spaces associated to compact hyperbolic manifolds, in Discrete Groups in Geometry and Analysis, papers in honor of G. D. Mostow on his sixtieth birthday, Progress in Math. 67 (1987), 48-106.

[KaL1] M. Kapovich and B. Leeb, On asymptotic cones and quasi-isometry classes of fundamental groups of 3 -manifolds, Geom. and Funct. Anal., 5 (1995), 582-603.

[KaL2] M. Kapovich and B. Leeb, Quasi-isometries preserve the geometric decomposition of Haken manifolds, Invent. Math. 128 (1997), 393-416.

[KL] B. Kleiner and B. Leeb, Rigidity of quasi-isometries for symmetric spaces of higher rank, Inst. Hautes Études Sci. Publ. Math., to appear.

[KR] A. Koranyi and H. M. Reimann, Foundations for the theory of quasiconformal mappings on the Heisenberg group, Adv. in Math. 111 (1995), 1-87.

[Me] G. Mess, The Seifert conjecture and groups which are coarse quasi-isometric to planes, preprint, 1987.

[Mi] J. Milnor, A note on curvature and fundamental group, J. Diff. Geom. 2 (1968), 1-7.

[Mo1] G.D. Mostow, Strong rigidity of locally symmetric spaces, Ann. of Math. Studies, 78, Princeton Univ. Press, 1973.

[Mo2] _ Quasiconformal mappings in $n$-space and rigidity of hyperbolic space forms, Publ. I.H.E.S., Vol. 33, 1968.

[Pa] P. Pansu, Metriques de Carnot-Caratheodory et quasiisometries des espaces symmetriques de rang un, Ann. of Math. 129 (1989), 1-60.

[Ri] E. Rieffel, Groups coarse quasi-isometric to $\mathbb{H}^{2} \times \mathbb{R}$, Ph.D. thesis, UCLA, 1993.

[S1] R. Schwartz, The quasi-isometry classification of rank one lattices, Inst. Hautes Études Sci. Publ. Math., 82 (1995), 133-168 (1996).

[S2] _ Quasi-isometric rigidity and diophantine approximation, Acta Math. 177 (1996), 75-112.

[St] J. R. Stallings, On torsion-free groups with infinitely many ends, Ann. of Math. 88 (1968), 312-334.

[Su] D. Sullivan, On the ergodic theory at infinity of a discrete group of hyperbolic motions, in Riemann Surfaces and Related Topics, I. Kra and B. Maskit, eds., Ann. of Math. Studies, 97, Princeton Univ. Press, 1981, pp. 465-496.

[Th] W. P. Thurston, The geometry and topology of three-manifolds, Princeton University Lecture Notes.

[Tu1] P. Tukia, On quasiconformal groups, J. D'Analyse Math. 46 (1986), 318-346.

[Tu2] _ Homeomorphic conjugates of Fuchsain groups, J.f.r.u.a. Math. 391 (1988), $1-54$.

[Tu3] Convergence groups and Gromov's metric hyperbolic spaces, New Zealand J. Math. 23 (1994), 157-187.

[Tu4] Q Quasiconformal extension of quasisymmetric mappings compatible with a Möbius group, Acta Math. 154 (1985), 153-193.

Math Department, University of Chicago, 5734 University Ave., Chicago, Il 60637

E-mail address: farb@math.uchicago.edu 